\section{Prevalence of Adverse Drug Events in Long Term Care: Variations in Screening Between Nurses and Physician-Pharmacist Reviewers}

\author{
Jean F Kozak ${ }^{1,2,3,4 *}$ and Akber Mithani ${ }^{5,6,7}$ \\ ${ }^{1}$ Centre for Health Evaluation and Outcome Sciences, University of British \\ Columbia, Vancouver, British Columbia, Canada \\ ${ }^{2}$ Department of Community and Family Medicine, Primary Care of the \\ Elderly Research Group, Providence Health Care, Vancouver, British \\ Columbia, Canada
}

${ }^{3}$ School of Population and Public Health, University of British Columbia, Vancouver, British Columbia, Canada

${ }^{4}$ The Centre for Education and Research on Aging \& Health (CERAH), Lakehead University, Ontario, Canada

${ }^{5}$ Department of Family and Community and Family Medicine, Mount St. Joseph's Elder Care Program, Providence Health Care, Vancouver, British Columbia, Canada

${ }^{6}$ Department of Psychiatry, University of British Columbia, British Columbia, Canada

${ }^{7}$ Department of Family Practice, University of British Columbia, British Columbia, Canada

\begin{abstract}
Screening for Adverse Drug Events (ADEs) in nursing home setting is a crucial component of appropriate, quality care. This study examined potential differences in screening for ADEs by nurses and a physician-pharmacist consensus panel. A modified Harvard Medical Practice Study protocol was used in a two-stage screening process to identify ADEs in a retrospective chart review of 134 patients across four care facilities. The study nurse chart reviewers identified 17 of the 134 patients as having experienced an ADE. The total number of incidents identified among the 17 patients was 20. The two most frequent types of nurse identified ADEs were drug reactions $(26.7 \%)$ and medication errors $(10.0 \%)$. In contrast,
\end{abstract}

*Corresponding author: Jean F Kozak, Centre for Health Evaluation and Outcome Sciences, Providence Health Care, 1081 Burrard Street, Vancouver, British Columbia, Canada, Tel: +1 6047822746; Fax: +1 6048068005; E-mail: jkozak@providencehealth.bc.ca

Citation: Kozak JK, Mithani A (2015) Prevalence of Adverse Drug Events in Long Term Care: Variations in Screening Between Nurses and Physician-Pharmacist Reviewers. J Gerontol Geriatr Med 1: 007.

Received: August 13, 2015; Accepted: November 18, 2015; Published: December 03, 2015 the consensus panel reviewing the same nurse identified 17 patients identified a total of $69 \mathrm{ADE}$ incidents. The largest discrepancy between the two screening groups was in the identification of adverse drug reactions (8 versus 46) and untreated conditions (none versus 7 ). The results underscore the need for further research examining how health care professionals use their clinical knowledge in identifying ADEs, and the development of protocols that address such possible professional differences.

\section{Introduction}

Older adults are the most rapidly expanding age group in North America and elsewhere. According to a 2013 United Nations report [1], the number of persons aged 60 and older is projected to double from 841 million to more than 2 billion in 2050, representing $21.1 \%$ of the world's population. Although many older adults lead healthy and active lives, a significant number suffer with multiple chronic health problems that limit their activities and require a wide spectrum of social and health services [2,3]. This increase in comorbid conditions has resulted in higher medication use among older adults. In a recent 2010 report from the Canadian Institute on health information analyzing pharmaceutical data from 2002 to $2008,21 \%$ of older adults were reported as using 10 or more types of prescription drugs with $6 \%$ using 15 or more drugs [4]. Similar trends have been reported in the United States as elsewhere $[5,6]$.

Concomitant with the increase in prescribed medications is the risk for adverse events among older adults. In a review of 15,000 hospitalizations in the United States, Thomas and Brennan [7] reported that the incidence rates for an adverse event nearly doubled for patients 65 years of age and older as compared to younger patients (5.29\% versus $2.80 \%)$ with preventable adverse event rates showing a similar age-related patent (2.95\% versus $1.58 \%)$. Similar and higher trends for older adults have been reported in the Canadian and International literature as well [8-10].

Although drug related issues among older adults, such as polypharmacy, has been well researched, there have relatively been only a small number of research prevalence studies in North America outside of the community and acute care sectors that focus on medication-related adverse events in the Long Term Care sector (LTC) [11-15].

Gurwitz and colleagues [15] examined the incidence of Adverse Drug Reactions (ADRs) in two large academic LTC facilities (one Canadian and one American) and reported that over a 9-month period, the ADR rate was 9.8/100 resident-months with a rate of 4.1 preventable ADRs per 100 resident-months. As expected, residents prescribed multiple drugs were more likely to experience preventable ADRs. Similar findings have been reported in the United States and elsewhere [16-17].

Baker et al., [9] examined the rate of adverse events in a variety of teaching and community acute care hospitals across Canada and found that overall; the incidence rate for overall adverse events was 7.5 per 1,000 cases, with $36.9 \%$ of the cases judged to have been preventable. With regard to older adults in acute care, the authors reported that older patients were more likely to experience AEs than 
younger aged patients. Similarly, a study conducted by Burgess et al., [18] found that older Australians experienced the greatest increase in AEs (7.7/1000 person years at ages $60-69$ to $34.3 / 1000$ person years at ages 80 and older). Similar trends have been reported for older adults elsewhere [11,19].

Given the expected increase of elders residing in long term care institutions and the fact that elders are more susceptible to adverse events than younger aged patients, and that there is a dearth of epidemiological studies to substantiate and explore the prevalence of adverse drug events among older adults within the long term care sector, we conducted this study to address the gap in our knowledge of adverse events among older adults residing in LTC settings. The primary objectives of the study was to determine the overall prevalence rate of Adverse Drug Events (ADEs) in long term care and to explore potential variations in prevalence due to who is conducting the screening.

\section{Methods}

A semi-randomized, retrospective chart review process was undertaken to identify adverse drug events in residents residing in four medium to large sized long term care facilities in Vancouver, British Columbia, Canada. In addition, incident data reports were obtained from all of the four study sites paralleling the same 12-month time period of the chart review component. Based on Baker et al.,s prevalence rate for AEs and that of Gurwitz et al.,'s for medication AEs in LTC, it was estimated that 134 charts across the four participating sites would be sufficient to detect an adverse event with a confidence level of $95 \%$ and an error margin of $5 \%$.

For the chart review, ADEs were defined as any unwanted or negative adverse effect(s) associated with a medication. An ADE could consist of any of the following events: untreated medical condition requiring medication, such as depression with no prescribed anti-depressant; wrong drug administered; subtherapeutic and supratherapeutic dose; drug interactions resulting in a medical condition; drug administered without indication; and an adverse drug reaction (for example, a fall associated with a medication).

At the start of the study, a list of residents residing at each of the four sites was generated. In order to be qualified for selection, the most recent month under retrospective review had to be at least 6-months prior to any current care underway. This criterion ensured that no chart review could possibly influence current care as well as ensuring the access to archived charts. One hundred and thirty-four patients who met this inclusionary criterion were selected through a computer program that randomly generated patient chart numbers stratified by site. These charts were then reviewed through Baker et al.,'s two-stage method and was modified to clinical procedures typically done in LTC settings. Items not relevant to older adults (e.g., abortion) were excluded, and those pertinent to them (e.g., abuse and neglect, bed pressure sores) were added. Charts were reviewed in monthly segments for the 12-month study period.

Prior to conducting the study, the chart study teams underwent training using the modified Baker protocol for screening and identifying adverse events from patient charts. There were two chart study teams: 1) nurse team consisting of four LTC RNs (clinical nurse specialists); and 2) a consensus review team consisting of two physicians and a $\mathrm{PhD}$ pharmacist. All of the chart review members had extensive experience working in LTC settings. Each group, upon completion of training, were required to independently review the same 5 sets of patient charts randomly selected from the participating sites that were not included in the main chart review. Disagreements between raters, regardless of team, were reviewed and definitions were clarified. Analyses of rater agreement across both groups ranged from an average Kappa [20] of 0.875 on basic demographic information such as age, length of hospital stay, medical history, and laboratory test results to 0.60 seen in those areas where clinical judgment was required to make a decision, excluding AEs, such as disease progression and health status.

In stage 1 of the chart review process, each nurse was provided with the randomized list that they were required to review independently of one another. The nurse reviewers recorded basic demographic information, and then screened each month in the charts for actual/potential adverse events as outlined in the modified LTC protocol. The reviews also included the monthly medication reports for that time period as well as nursing notes and any consultation reports.

In stage 2, the nurse identified $\mathrm{ADE}$ incidents were then examined by the clinical consensus team (team \#2) consisting of two physicians and pharmacist. The consensus team first reviewed each nurse identified incident independently of one another, and then met at a consensus conference where each identified event was discussed and rated on: 1) the likelihood that the event was drug related; 2) how preventable the event could have been; and 3) the level of severity of the event to the patient, ranging from no evident effect to death. The Strand protocol was used to assist in identifying potential drug adverse events likelihood and outcomes. In addition to the nurse identified ADEs, the full 12-month charts of these nurse-screened patients were also reviewed by the consensus team to determine if these same patients were at risk for other ADEs not identified by the nurse screeners. All chart reviewers were blind to facility level reports on medication incidents for the same time period reviewed.

The data recorded by the two teams of reviewers were entered into IBM SPSS Version 20.0 and analyzed. Tests of significance conducted were two-tailed. The study was approved by the joint University of British Columbia and Providence Health Care Research Ethics Board.

\section{Results}

The chart reviews were equally distributed among the four study sites ranging from $15 \%$ at site 3 to $33.7 \%$ at site 4 . Overall the reviewed charts were done on $21.9 \%$ of 611 patients across all of the sites. Of the 134 patients whose charts were reviewed, the majority (Table 1 ) were female ( 89 or $66.4 \%$ ), and ages ranged from 65 years to 104 years (average age 82.99 years; $\mathrm{SD}=8.89$ ). Analysis of variance indicated that there were no significant age differences across the four sites $(\mathrm{F}=1.99, \mathrm{df}=3, \mathrm{p}>.05)$ but that women overall were significantly older than men $(\mathrm{F}=8.86, \mathrm{df}=1, \mathrm{p}=.003)$. There were significantly more women than men across the study sites $\left(\chi^{2}=52.3, \mathrm{df}=3, \mathrm{p}<.001\right)$.

Just under one half of the patients (47.8\%) had a length of stay of two or more years, followed in frequency by 1-2 years (15.7\%), 6 months - 1 year (14.9\%) and 3-6 months (6.7\%). Twenty of the 134 patients had a length of stay of less than three months (14.9\%). There was a significant sex difference in length of stay $\left(\chi^{2}=10.42, \mathrm{df}=4\right.$, $\mathrm{p}=0.034$ ), with women having proportionally more individuals with a length of stay greater than six months.

The top 10 comorbidities, shown in table 2, range from cardiovascular disease (41.8\%) and non-Alzheimer's dementia (37.3\%) to seizure disorders (10.4\%). Of these, the dementias (Alzheimer's and 


\begin{tabular}{|c|c|c|c|c|}
\hline & & Female & Male & p level \\
\hline $\begin{array}{c}\text { Frequency } \\
\text { (Percent) }\end{array}$ & $89(66.4 \%)$ & $45(33.6 \%)$ & $<0.001$ \\
\hline Age: & Mean & 84.6 years & 79.9 years & $<0.01$ \\
\hline & Standard Deviation & 8.71 years & 8.48 years & \\
\hline $\begin{array}{c}\text { Length of Stay } \\
\text { (frequency) }\end{array}$ & Less than 3 months & 11 & 9 & $<0.05$ \\
\hline & 3 to 6 months & 6 & 3 & \\
\hline & 6 to 12 months & 19 & 1 & \\
\hline & 1 to 12 years & 11 & 8 & \\
\hline & More than 2 years & 41 & 23 & \\
\hline
\end{tabular}

Table 1: Sample Demograhics.

non-Alzheimer's combined) form the most prevalent comorbid condition $(n=73,57.4 \%)$. The average number of comorbid conditions was 5.1 , with men having slightly more comorbid conditions (mean $=6.2)$ than female patients (mean $=4.6)$. These differences were not significant at the .05 level $(t=1.663, \mathrm{df}=132, \mathrm{p}>0.05)$. There were no significant differences across the four study sites on comorbidity count $(\mathrm{F}=1.087, \mathrm{df}=3, \mathrm{p}>.05)$. At completion of the reviews, 121 patients were still alive and residing at the facility (90.3\%); 10 had died (7.5\%); 2 had been transferred to acute care (1.5\%); and 1 had been discharged to another facility $(0.7 \%)$. None of the deaths among the reviewed patients were related to an adverse drug event.

\begin{tabular}{|c|c|c|}
\hline Diagnosis & Frequency & Percent \\
\hline Cardiovascular & 56 & $41.80 \%$ \\
\hline Dementia: Non Alzheimer's Disease Depression & 46 & $37.30 \%$ \\
\hline Depression & 34 & $25.40 \%$ \\
\hline Type 2 Diabetes & 33 & $24.60 \%$ \\
\hline Alzheimer's Disease & 27 & $20.10 \%$ \\
\hline Osteoarthritis & 25 & $18.70 \%$ \\
\hline Osteoporosis & 21 & $15.70 \%$ \\
\hline Allergies & 20 & $14.90 \%$ \\
\hline Hemiplegia/Hemiparesis & 17 & $12.70 \%$ \\
\hline Seizure Disorders & 14 & $10.40 \%$ \\
\hline All Dementias (Alzheimer+Non) & 73 & $57.40 \%$ \\
\hline \multicolumn{2}{|c|}{ Table 2: Ten most frequent comorbid diagnosis. } \\
*Diagnoses are not independent and therefore do not sum to 100\% \\
\hline
\end{tabular}

Of the 134 patients screened by the nurse reviewers, 20 incidents (14.9\%) involving 17 patients were identified as possibly being an ADE (Table 3). The study nurse identified ADE incident rate of $14.0 \%$ was comparable with the facility level reported rate of $16.1 \%$ for the same time period in question. The most commonly identified ADE by the nurse reviewers were drug reactions ( 8 out of 20 incidents) involving rashes associated with L-Tryptophan and Clavulin. The second most frequent were medication errors $(n=2)$ due to the administration of the wrong drug dose. Seven of the twenty incidents were suspected ADEs, but were unable to be classified by the nurse reviewers as an adverse event. These suspected cases, and the remaining three identified incidents shown in table 3 , involved a variety of medications ranging from Amoxicillin to Amitripltine and Resperidone, with no medication having more than one incident associated with it.

The physician-pharmacist consensus team (Table 3 ) identified a total of 69 incidents within the 12-month period for the same 17

\begin{tabular}{|c|c|c|}
\hline Screener & Nurses & Consensus Team \\
\hline Untreated Condition & - & 7 \\
\hline Adverse Drug Reaction & 8 & 46 \\
\hline Medication Error & 2 & 2 \\
\hline Subtherapeutic Dose & 1 & 6 \\
\hline Supratherapeutic Dose & 1 & 3 \\
\hline Drug-Drug Interactions & 1 & 5 \\
\hline Not an ADE & 7 & 2 \\
\hline Total & 20 & 69 \\
\hline
\end{tabular}

Table 3: Adverse drug event classification frequency by screener for 17 patients.

patients that were identified as having had an ADE (20 incidents). The consensus team was in agreement with 13 of the ADE incidents identified by the nurse chart reviewers, but felt that 7 of the suspected incidents were not medication related. Among the consensus team, two potential incidents were excluded as no consensus could be obtained on whether the incidents were a drug-to-drug interaction or the result of a disease progression.

Of the 69 ADEs agreed upon by the consensus team, the top three classifications as shown in table 3 are adverse drug reactions $(n=46)$, untreated conditions $(n=7)$, and subtherapeutic doses $(n=6)$. As seen in the table 3, the study nurses and physician-pharmacist consensus team differed in the number of identified adverse events, the largest being in the areas of adverse drug reactions ( 8 incidents for nurses versus 46 for the consensus team) and untreated conditions (none versus 7). The most frequent consensus team identified ADE were bleeds and anemia $(\mathrm{n}=10)$ associated with the administration of Aspirin when a patient was on Warfarin, followed by abnormal laboratory results $(\mathrm{n}=6)$ associated with medications such as Dilantin and Carbamazepine. Five ADEs were associated with anti psychotic medications administered during ECT. Gastrointestinal disorders, involving nausea, vomiting, constipation and diarrhea, were the next most frequent involving four cases and were associated with medications such as Citalopram and Furosemide. Three ADE incidents identified by the consensus team were classified for each of the following signs and symptoms: headache; cardiac symptoms; falls; sedation; somnolence; and seizures. Medication errors due to the administration of wrong doses were the last most frequent ADE classification $(\mathrm{n}=2)$. The remaining 24 consensus team identified ADEs were events associated with unique medications and clinical or laboratory signs, such as hypokalemia associated with the use of a diuretic (e.g., Furosemide).

As shown in table 4 , the consensus team classified $71.7 \%$ of the incidents as having moderate to strong evidence, and $2.5 \%$ as having little or no evidence. Only $1.7 \%$ of the incidents were not deemed to be preventable, whereas $72.5 \%$ of the incidents were seen as having strong or better evidence for being preventable. Of the consensus team identified incidents: $11.7 \%$ had no associated disability or impairment; $65.8 \%$ had minimal impairment (improvement within one month); $12.5 \%$ moderate impairment with recovery less than 6 months and $6.7 \%$ moderate impairment with recovery within 6 to 12 months. There were no cases of death or acute care hospitalization associated with the ADE. Four cases (3.3\%) could not be classified by the consensus team as to level of associated disability due primarily to disease progression. 


\begin{tabular}{|c|c|c|}
\hline Level of Evidence & $\begin{array}{c}\text { Management } \\
\text { Causation }\end{array}$ & Preventabiliy \\
\hline Virtually no evidence & $1.7 \%$ & $1.7 \%$ \\
\hline Less likely (but less than 50/50) & $0.8 \%$ & - \\
\hline More likely (more than 50/50) & $16.7 \%$ & $25.8 \%$ \\
\hline Moderate to strong evidence & $71.7 \%$ & $66.7 \%$ \\
\hline Virtually certain evidence & $9.2 \%$ & $5.3 \%$ \\
\hline
\end{tabular}

Table 4: Adverse Drug Event Classification by Level of Evidence for Management Causation and Possible Prevention.

\section{Discussion}

The results of the study indicate that a large majority of ADEs may be under reported and that the screening for them may differ across professional lines even when a standardized protocol is used. The study nurse screeners identified almost similar numbers of adverse drug events over the four study sites similar to those reported independently at the facilities for the same time period (14.9\% versus $16.1 \%)$, rates that are within the ranges $(9.8 \%$ to $19.4 \%)$ of those reported in previous literature on ADEs in this population [8,13-16].

In contrast, the consensus panel of two physicians and a pharmacist detected more than 3 times the number of ADE incidents in the same 17 patients when reviewing the 12-month period. The areas in which the discrepancy between the nurse screeners and those of the consensus team are the largest is that of adverse drug reactions, dosage (sub and supratherapeutic ranges) and drug to drug interactions. This finding appears to suggest that, although the same protocol was being used by both groups to screen for ADEs, each group may have brought their different clinical training into the decision of what event may have been a suspected ADE. This can be seen in the detection of adverse drug events and untreated conditions, which requires an in-depth knowledge of pharmacodynamics, clinical signs and symptoms, and laboratory results: training that tends to be more comprehensive for physicians and pharmacists than nurse practitioners in Canada as elsewhere.

There has been a growing emphasis on the urgent need for developing standardized protocols for the screening of ADEs, particularly among high risk groups as older adults [21,22]. Recent attempts have focused on the use of electronic reporting systems that are assumed to reduce reporting errors while improving surveillance [22]. Others have advocated the use concurrent monitoring by a clinical team whereas others have emphasized the importance of pharmacists in conducting ADE surveillance due to their level of training [23,24]. Regardless of method employed, the results of this study suggests that we need to have a better understanding of how professional groups like nurses, physicians, and pharmacists use their clinical knowledge and experience in detecting a possible ADE. A related issue was raised by Shmueli et.al., [25] who reported that older nurses reported more ADEs in a nursing home setting than did younger aged nurses. This and the present study indicate that great care is needed in designing both studies and reporting/monitoring procedures that use primarily one professional group over another. It is possible that nurses, physicians, and pharmacists may be using different criteria, ones based on experience and professional training, when screening for an ADE. For example, physicians and pharmacists may have greater knowledge of drug-drug interactions and therefore may be more likely to identify such events than regular nursing staff.

Equally of concern is the increasing reliance on non-RN nursing staff in the nursing home sector. There has been extensive discussion among clinicians regarding how bedside care is increasingly being provided by less clinically skilled care staff due to rising costs, a trend that raises issues of the ability of such staff in detecting more subtle clinical changes due to illness or iatrogenic causes. Care must be undertaken to develop methodologies that optimize the contribution of each professional group while reducing potential biases that can dramatically affect the reported prevalence and incidence of ADEs.

None of the identified ADEs in this study resulted in a serious reportable event (death or hospitalization) as defined by health authorities such as Health Canada; and, therefore, would not likely be identified as such in clinical quality assurance reports. Although not reportable per se, what is unknown is whether such subthreshold reportable events may, if continued, result in an actual definable serious event. Future research is needed to explore the possible relationship between cumulative adverse drug events and poor health outcomes.

Adverse drug events among a highly frail population is a highly complex problem requiring a multi pronged approach that must address numerous complex issues including education, training and certification of clinical staff; standardization of definitions of a medication adverse event (subthreshold and threshold); standardization of incident review and monitoring processes; and standardization of medical charting to ensure documentation of suspected and actual events. Adverse drug events are not just a function of age alone, but the interaction of physiological and functional characteristics of a patient at a specific time in his or her comorbid disease(s). The detection and reporting of adverse events associated with the ongoing changes in medication typically found in such a frail patient population requires an understanding of these myriad of issues and the involvement of various clinical skill sets. Further research is needed on how clinicians decide what constitutes an ADE in such complex, dynamic interactions in older, more frail patients as well as how to standardize such processes in a health setting where bedside care is becoming more reliant on less skilled clinical staff.

\section{Study Limitations}

Because of the Baker protocol used, which captures only the nurse and combined consensus team data, the study was unable to explore specific nurse, physician, and pharmacist variations in identifying adverse events. A separate study is underway exploring physician versus nurse variation in detecting drug and non drug related ADEs. Although the current study had sufficient sample size to detect an adverse event, studies with larger sample sizes are needed to explore specific types of adverse drug events, the class and type of medications associated with those events, and their associated patient outcomes. A similarly larger study is also needed to explore possible ADE incident variation due to the type of signs and symptoms for which a patient being treated, for example, comparing cardiovascular disorders among patients with and without difficult to manage behaviors.

\section{Conclusion}

The causes and detection of an adverse drug event is a complex process requiring training, clinical and pharmaceutical knowledge, and sensitive surveillance. The findings indicate that even with the use of clinicians trained with a standardized adverse incident tool, variations in professional knowledge and practice may lead to differing rates of detection. More research on how clinicians view and identify an adverse event among a frail older population vulnerable to such events is needed. 
Citation: Kozak JK, Mithani A (2015) Prevalence of Adverse Drug Events in Long Term Care: Variations in Screening Between Nurses and Physician-Pharmacist Reviewers. J Gerontol Geriatr Med 1: 007.

\section{Acknowledgment}

This study was funded through a grant from the Canadian Patient Safety Institute.

\section{References}

1. United Nations (2013) World Population Ageing 2013. ST/ESA/SER.A/348. Department of Economic and Social Affairs, Population Division. United Nations, New York, USA.

2. Wolff JL, Starfield B, Anderson G (2002) Prevalence, expenditures, and complications of multiple chronic conditions in the elderly. Arch Intern Med 162: 2269-2276.

3. Marengoni A, Angleman S, Melis R, Mangialasche F, Karp A, et al. (2011) Aging with multimorbidity: a systematic review of the literature. Ageing Res Rev 10: 430-439.

4. Canadian Institute for Health Information (2011) Seniors and the health care system: what is the impact of multiple chronic conditions? Canadian Institute for Health Information, Canada.

5. World Health Organization (2005) Facing the facts: the impact of chronic disease in Canada. Preventing chronic diseases: a vital investment. World Health Organization, Geneva, Switzerland.

6. Mueller C, Schur C, O'Connell J (1997) Prescription drug spending: the impact of age and chronic disease status. Am J Public Health 87: 1626-1629.

7. Freund D, Smeeding TM (2010) The future costs of health care in ageing societies: Is the glass half full or half empty? In: Tuljapurkar S, Ogawa N, Gauthier AN (eds.). Ageing in advanced industrial states: Riding the age waves, Vo 3. Springer Science \& Business Media, Heidelberg, Germany 3: 173-193.

8. Thomas EJ, Brennan TA (2000) Incidence and types of preventable adverse events in elderly patients: population based review of medical records. BMJ 320: 741-744.

9. Baker GR, Norton PG, Flintoft V, Blais R, Brown A, et al. (2004) The Canadian Adverse Events Study: The incidence of adverse events among hospital patients in Canada. CMAJ 170: 1678-1686.

10. Gerety MB, Cornell JE, Plichta DT, Eimer M (1993) Adverse events related to drugs and drug withdrawal in nursing home residents. J Am Geriatr Soc 41: $1326-1332$.

11. Midlöv P, Bergkvist A, Bondesson A, Eriksson T, Höglund P (2005) Medication errors when transferring elderly patients between primary health care and hospital care. Pharm World Sci 27: 116-120.

12. Daneman N, Bronskill SE, Gruneir A, Newman AM, Fischer HD, et al. (2015) Variability in Antibiotic Use Across Nursing Homes and the Risk of Antibiotic-Related Adverse Outcomes for Individual Residents. JAMA Intern Med 175: 1331-1339.
13. Handler SM, Wright RM, Ruby CM, Hanlon JT (2006) Epidemiology of medication-related adverse events in nursing homes. Am J Geriatr Pharmacother 4: $264-272$

14. Perri M 3rd, Menon AM, Deshpande AD, Shinde SB, Jiang R, et al. (2005) Adverse outcomes associated with inappropriate drug use in nursing homes. Ann Pharmacother 39: 405-411.

15. Gurwitz JH, Field TS, Judge J, Rochon P, Harrold LR, et al. (2005) The incidence of adverse drug events in two large academic long-term care facilities. Am J Med 118: 251-258.

16. Butler M, Norton R, Lee-Joe T, Cheng A, Campbell AJ (1996) The risks of hip fracture in older people from private homes and institutions. Age Ageing 25: $381-385$

17. Thapa PB, Gideon P, Cost TW, Milam AB, Ray WA (1998) Antidepressants and the risk of falls among nursing home residents. N Engl J Med 339: 875882.

18. Burgess CL, Holman CD, Satti AG (2005) Adverse drug reactions in older Australians, 1981-2002. Med J Aust 182: 267-270.

19. Boockvar K, Fishman E, Kyriacou CK, Monias A, Gavi S, et al. (2004) Adverse events due to discontinuations in drug use and dose changes in patients transferred between acute and long-term care facilities. Arch Intern Med 164: 545-550.

20. Gwet K (2002) Computing Inter-rater reliability with the SAS system. Series: Statistical Methods for Inter-Rater Reliability Assessment no:3. Pg no: 1-16.

21. Lapane KL, Hughes CM (2004) Identifying nursing home residents at high risk for preventable adverse drug events: modifying a tool for use in the Fleetwood Phase III Study. Consult Pharm 19: 533-537.

22. Handler SM, Hanlon JT (2010) Detecting Adverse Drug Events Using a Nursing Home Specific Trigger Tool. Ann Longterm Care 18: 17-22.

23. Boockvar KS, Carlson LaCorte H, Giambanco V, Fridman B, Siu A (2006) Medication reconciliation for reducing drug-discrepancy adverse events. Am J Geriatr Pharmacother 4: 236-243.

24. Crotty M, Rowett D, Spurling L, Giles LC, Phillips PA (2004) Does the addition of a pharmacist transition coordinator improve evidence-based medication management and health outcomes in older adults moving from the hospital to a long-term care facility? Results of a randomized, controlled trial. Am J Geriatr Pharmacother 2: 257-64.

25. Shmueli T, Noy RH, Ben Natan M, Ben-Israel J (2014) Reporting adverse events at geriatric facilities: categorization by type of adverse event and function of reporting personnel. Int J Health Care Qual Assur 27: 91-98. 